\title{
ULTRASOUND DIAGNOSTICS AT THE STAGES OF EXTRACORPOREAL RESECTION OF A SINGLE KIDNEY IN THE TREATMENT OF RENAL CELL CARCINOMA
}

\author{
Yulia A. Stepanova, Vlada Yu. Raguzina*, Tatiana P. Baitman, \\ Olesya A. Chekhoeva, Irina V. Miroshkina, Aleksandr A. Gritskevich
}

\author{
A.V. Vishnevsky National Medical Research Center of Surgery, Moscow, Russia
}

\begin{abstract}
An organ-sparing approach is preferable at the treatment of patients with cancer of a solitary kidney, but doesn't always comply with the oncological radicalism. The technique of extracorporeal renal resection followed by autologous transplantation was developed to preserve renal function in patients with obligatory indications for organpreserving treatment. The aim is to evaluate the possibilities of ultrasound (US) at the stages of extracorporeal resection of a single kidney in the treatment of renal cell carcinoma. Materials and methods. The study included 22 patients treated with renal cell carcinoma of a single kidney in 2013-21 (average age 60.45 \pm 7 years). Men prevailed (73\%). Multiple primary metachronous cancer occurred in 16 (73\%) cases, multiple primary synchronous cancer - in 2 (9\%), previous nephrureterectomy was performed in connection with benign kidney diseases (primary contracted kidney, hydronephrosis) - in 2 (10\%), a congenital single kidney was in 2 (10\%) patients. Previously underwent surgery on a single kidney for a malignant neoplasm of the same etiology for which 6 (27\%) patients are being treated in this hospitalization. All the patients underwent US examination in B-mode and duplex scanning at the pre-/intra-and postoperative stage. If necessary, echo-contrast US (Sonovue) was performed intraoperatively and in the early postoperative period. Also, all patients underwent preoperative contrast-enhanced multidetected computed tomography (MDCT). MRI was performed in 7 cases. All the patients were operated with histological verification. Results. Staging according to the TNM system: pT1a-T3vNo-2Mo-1G1-3, of which the tumor size exceeded $7 \mathrm{~cm}$ in $1 O$ (50\%) patients, distant metastases were in 8 (40\%) cases. Reno-caval tumor thrombus was detected in 3 patients. Intraoperative US was performed at the stages of surgery: navigation to the stage of resection and assessment of the restoration of blood supply in the intervention area after kidney resection and wound closure. In 3 cases, extracorporeal renal resection was performed simultaneously with thrombectomy and resection of the inferior vena cava for renocaval tumor thrombus. In 4 cases, renal vessel replacement was performed. The tumor involved vessels in 3 cases and in 1 IOUS after resection showed thrombosis of the renal artery, which eventually required prosthetics. There were no intraoperative complications. All patients underwent US-monitoring on the 1st, 3 rd and 5 th days after surgery, more often and further as needed. The follow-up period (US, MSCT) was 19-85 months (53.3 117.2). Tumor progression occurred in $3(15 \%)$ cases. One patient died due to the progression of the tumor process 20 months after the operation. Conclusion. US make it possible to control all the stages of extracorporeal resection of a single kidney under pharmacocold anti-ischemic protection with orthotopic replantation of renal vessels. The results of this surgical intervention are satisfactory, which indicates the advisability of further development of organ-saving treatment.
\end{abstract}

Key words: Tumour of a single kidney, Ultrasound diagnostics, Radiology, Renal cell carcinoma, Nephron-sparing surgery

\section{INTRODUCTION}

Renal cell carcinoma (RCC) takes the 10th place in the cancer morbidity pattern for both sexes. There is a significant $20 \%$ increase in RCC incidence over the last 10years [1].

One of the features of RCC is its frequent spread along the venous bed: the tumor spreads to the renal vein in $25-30 \%$ of cases; into the inferior vena cava - in $4-10 \%$; in the right atrium - in $1 \%$ of cases [2].

Surgical care still remains the main way to treat RCC. While nephron-sparing surgery (NSS) is extremely favoured and recommended for the RCC of IIII stage, if technically feasible [3], the main barrier for its wide adoption is a high risk of acute kidney injury and, afterwards, chronic kidney disease. Nowadays, there are several ways to protect the kidney function, such as pharmacological, cold or pharmaco-cold anti- ischemic methods, the methods of reduction the time or the area of warm ischemic injury.

RCC of a solitary kidney is a problem of particular complexity. The incidence of congenital unilateral renal state is about 1 in 1000 [4]. Acquired solitary kidney after a unilateral radical nephrectomy in adults is mainly due to living kidney donation, renal tumor, and trauma. Over the last 30 years, rate of living kidney donation in the world had gradually increased from 1800 donations in 1998 to 6600 donations in 2004 However, it has decreased since 2011 and has been stable around 5650 kidney donations per year. The world incidence of RCC is 63,990 cases each year. Due to the literature, there were 10,123 and 4299 radical nephrectomies, due to RCC, performed during 19912002 and 1992- 2007, respectively. The incidence of renal trauma is varied. One study reported 757 radical nephrectomies among 9002 renal trauma patients from 2002-2007 [5]. As usual, the renal function of patients

*vladalove94@bk.ru 
with RCC of the solitary kidney is already impaired before the treatment [5-7], which makes the NSS approach especially significant.

The method of extracorporeal renal resection under pharmaco-cold anti-ischemic protection with orthotopic vascular replantation is currently expanding the indications for their use. This method is used to perform organ-saving surgical treatment for central and intraparenchymal location of the tumor in the kidney; with tumors of large sizes, but potentially resectable; in patients with tumors of the only kidney; with bilateral neoplastic lesion of the kidneys; in patients with concomitant kidney disease, both neoplastic and nonneoplastic. The surgical technique used at A.V. Vishnevsky National Medical Research Center (NMRC) of Surgery differs from similar techniques in other surgical clinics in the absence of the need for heterotopic autotransplantation of the kidney into the pelvic cavity (after the stage of extracorporeal resection, the kidney is returned to the retroperitoneal space in an orthotopic position with orthotopic replantation of renal vessels, which excludes the implementation of additional surgical access, which significantly reduces the duration operations) and the absence of the need for transection of the ureter with its subsequent stenting [8]. We prefer the method of pharmaco-cold antiischemic protection with the use of refrigerated $(+2-$ $4^{\circ} \mathrm{C}$ ) pharmacoplegic solution and ice crumbs. We perform partial nephrectomies from the extracorporeal surgical approach for large centrally localized, R.E.N.A.L. score 10-12, tumours of solitary kidneys. So, there are strict indications for this complex operation in our practice.

An important aspect of patient management during this surgical intervention, especially in the case of a single or only functioning kidney, is careful monitoring of the kidney condition at all stages of treatment. However, the only kidney and the possibility of developing renal failure do not allow to use the multidetected computed tomography (MSCT) with nephrotoxic contrast agent for monitoring. The need to perform control intraoperatively and in the early postoperative period (under resuscitation) makes it difficult to use magnetic resonance imaging (MRI) for these purposes. Thus, ultrasound (US) is the method of choice for monitoring the patient's condition at the stages of extracorporeal renal resection, so it is advisable to consider the effectiveness of using its modalities to assess various aspects of the patient's condition.

\section{AIM}

The aim is to evaluate the possibilities of US at the stages of extracorporeal resection of a single kidney in the treatment of RCC.

\section{MATERIALS AND METHODS}

The study included 22 patients treated with RCC of a single kidney in 2013-21 (average age 60.45 \pm 7 years). Men prevailed (73\%). Multiple primary metachronous cancer occurred in 16 (73\%) cases, multiple primary synchronous cancer - in 2 (9\%), previous nephrureterectomy was performed in connection with benign kidney diseases (primary contracted kidney, hydronephrosis) - in 2 (10\%), a congenital single kidney was in $2(10 \%)$ patients. Previously underwent surgery on a single kidney for a malignant neoplasm of the same etiology for which 6 (27\%) patients are being treated in this hospitalization. All the patients underwent US examination in B-mode and duplex scanning at the pre/intra- and postoperative stage. If necessary, echocontrast US (Sonovue) was performed intraoperatively and in the early postoperative period. Also, all patients underwent preoperative contrast-enhanced MDCT. MRI was performed in 7 cases. All the patients were operated with histological verification.

\section{RESUlts AND DisCUSSION}

Staging according to TNM system: pT1a-T3vNo2Mo-1G1-3, of which the tumor size exceeded $7 \mathrm{~cm}$ in 11 (50\%) patients, distant metastases were in 7 (32\%) cases. Reno-caval tumor thrombus was detected in 3 patients.

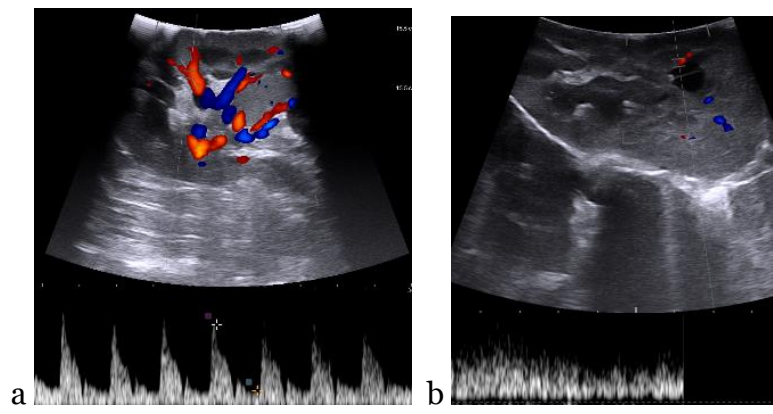

Figure 1. Duplex scanning of the kidney after suturing the resection area: $\mathrm{a}$ - normal blood flow; $\mathrm{b}$ - hemodynamically significant changes in blood flow
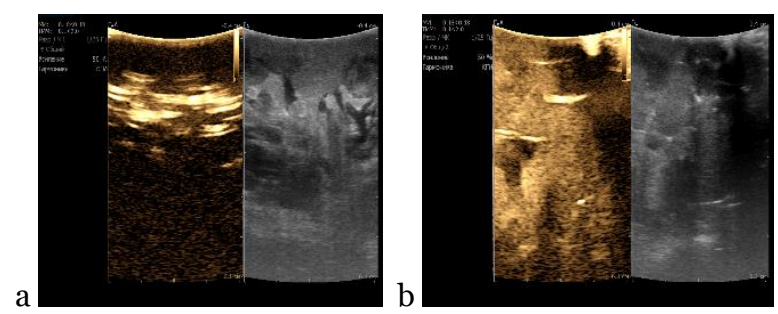

Figure 2. Restoration of intrarenal blood flow at echo-contrast IOUS: a - 10 sec., b - 25 sec.

Intraoperative US (IOUS) was performed at the stages of surgery: navigation to the stage of resection and assessment of the restoration of blood supply in the intervention area after kidney resection and wound closure (Fig. 1). Spasm of the microvasculature immediately after suturing the kidney in some cases did not allow correct assessment of the blood supply to the postoperative area at duplex scanning (there was no Color/Energy mapping of the vessels). In such cases, an echo-contrast US was performed, which made it possible to adequately assess the blood supply to this area (Fig. 2).

In 3 cases, extracorporeal renal resection was performed simultaneously with thrombectomy and 
resection of the inferior vena cava for reno-caval tumor thrombus. In 4 cases, renal vessel replacement was performed. The tumor involved vessels in 3 cases and in 1 IOUS after resection showed thrombosis of the renal artery, which eventually required prosthetics. The average duration of cold ischemia was $100.2 \pm 40.5 \mathrm{~min}$. Bloodloss $-500 \pm 150 \mathrm{ml}$. There were no intraoperative complications.

In the early postoperative period, in the case of acute renal failure, US was performed in duplex scanning mode and with echo-contrast. US duplex scanning is performed in the modes of Color and/or Power Doppler imaging (CDI/PDI) in order to confirm the presence or absence of intraorgan blood flow in the kidney. In the case of clinical anuria, intraorgan blood flow in the resected kidney is not always recorded. Next, US is performed with the echo-contrast (Fig. 3). The study was carried out in 6 out of 22 operated patients with anuria in the postoperative period. In all cases, the kidney was recognized as viable and the patients continued hemofiltration sessions until urine appeared. The general terms of anuria varied from 3 to 7-8 days.

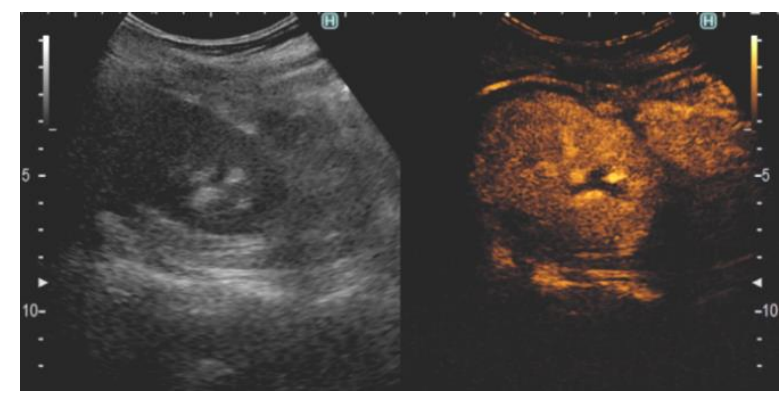

Figure 3. The patient after extracorporeal resection of a single kidney, anuria is noted on the 4th day after the operation. The vascular pattern is not determined at CDI/PDI. Echocontrast US shows restoration of intrarenal blood flow (34 sec.)

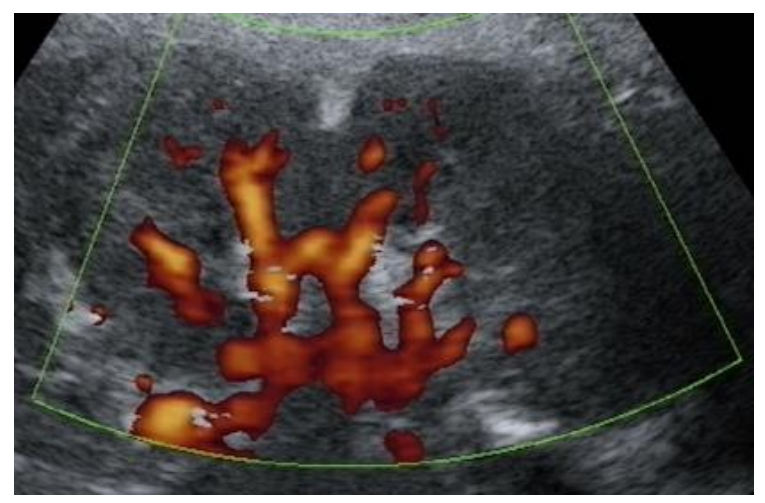

Figure 4. Vascular pattern of the kidney on the $5^{\text {th }}$ day after resection at PDI

All patients underwent US-monitoring on the 1st, 3 rd and $5^{\text {th }}$ days after surgery, more often and further as needed. The restoration of the vascular pattern of the kidney (Fig. 4), the state of the collecting system, the presence of hematoma, fluid accumulation or free fluid were assessed. Laboratory control of indicators was carried out also.

Postoperative complications $\geq$ grade II according to Clavien-Dindo classification were observed in 10 (50\%) patients: II - 1 (4.5\%), IIIa - 4 (18.2\%), IVa - 4 (18.2\%). Complications of IVa degree were characterized by the development of acute kidney injury, which required renal replacement therapy.

The follow-up period (US, MDCT) was 3-91 months (40.6 \pm 28.5$)$. Tumor progression occurred in 2 (9\%) cases. One patient died due to the progression of the tumor process 20 months after the operation.

Analysis of preoperative patients' examination data and their comparison with intraoperative findings and the result of postoperative monitoring showed that the following algorithm for patient examination is advisable.

\section{Preoperatively:}

- US in B-mode and duplex scanning with an assessment of the kidney, tumor, renal and inferior vena cava;

- MDCT/MRI of the kidney, tumor, renal and inferior vena cava with contrast enhancement (MDCT is preferred).

\section{Multistage US is performed intraoperatively:}

- to assess the localization of the tumor in the kidney, marking the contours of the tumor in its central/intramural localization;

- in the presence of an intraluminal thrombus in the renal/inferior vena cava, an US assessment of the thrombus in the vein is performed;

- after removal of the thrombus, the lumen of the vein is assessed;

- after resection and suturing of the kidney, the restoration of blood flow is assessed.

\section{Early postoperative period:}

- if it is difficult to visualize the intraorgan vascular pattern with US duplex scanning and/or anuria, it is advisable to perform US with contrast enhancement to assess the viability of the kidney;

- US-monitoring is carried out on the 1 st, 3 rd and $5^{\text {th }}$ days, more often and further as necessary.

\section{Long-term postoperative period:}

- US is performed every 6 months or when complaints appear;

- if a tumor recurrence or suspicious areas in the kidneys are detected, contrast-enhanced MDCT is performed.

In order to illustrate the application of this algorithm, we present a clinical case.

Patient B., 39 years old, had a single functioning right kidney. He complains of recurrent pain in the right lumbar region.

From the anamnesis it is known that an US scan at the place of residence revealed the lesion of the right kidney up to $5.0 \mathrm{~cm}$ in diameter. MDCT of the abdominal cavity and retroperitoneal space with contrast enhancement was performed on an outpatient basis at the place of residence, in which a tumor of a single kidney was diagnosed, measuring $5.0 \times 3.0 \mathrm{~cm}$, which had a predominantly extraorgan localization, intensively accumulating contrast agent. The patient was admitted to the Urology Department of A.V. Vishnevsky NMRC of Surgery for surgical treatment. 
In the lower segment of the right kidney, a rounded lesion of a heterogeneous structure with smooth, clear contours up to $5.4 \times 5.6 \times 5.7 \mathrm{~cm}$ was noted at MRI in the Center (Fig. 5a). The germination of the lower group of cups and the renal pelvis was determined. A hypointense soft tissue component in the lumen of the pelvis (thrombotic blood masses) was also determined in T1 and T2 WI (Fig. 5b). Conclusion. MR signs of right kidney tumor with invasion of the lower group of cups and pelvis. Thrombotic masses in the pelvis. Hypoplasia of the left kidney.

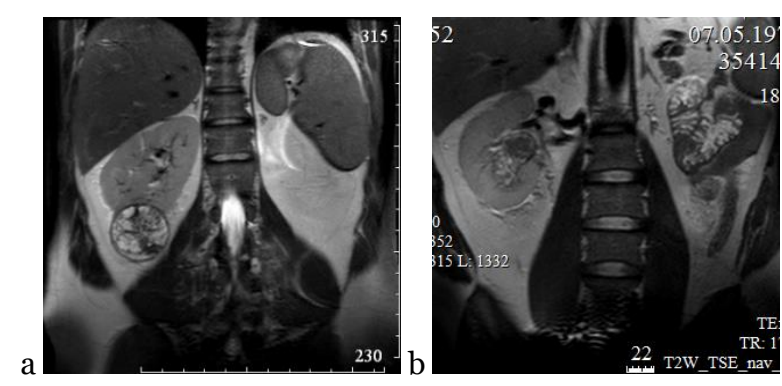

Figure 5. MRI, T2W_TSE: a - tumor of the lower segment of the right kidney; $\mathrm{b}$ - thrombotic masses in the pelvis

In order to assess the possibility of performing nephrostomy, an urgent US was performed. The pelvis of the only right kidney was filled with a blood clot. Also, minimum volume of the hemorrage was detected in the cups on the poles and a thin layer of relatively fluid blood at the level of the pelvic-ureteric junction. It was not possible to trace the ureter further. Conclusion. Subtotal hemotamponade of the collecting system of the only right kidney against the background of its tumor lesion.

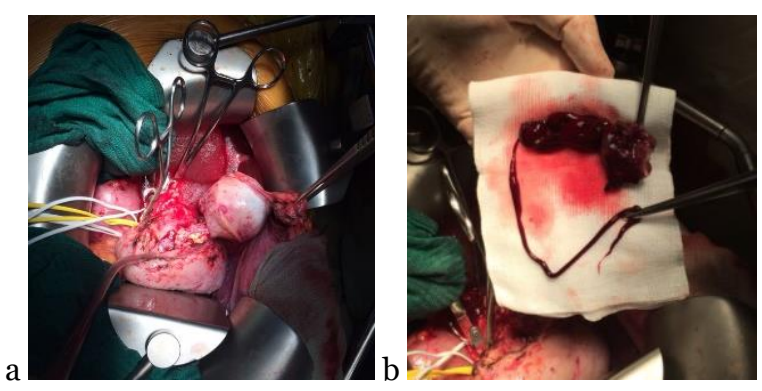

Figure 6. Intraoperative: a - stage of tumor removal; $\mathrm{b}$ - thrombotic masses removed from the pelvis

Taking into account the presence of ongoing bleeding, hemotamponade of the collecting system of the only right kidney, surgical intervention was performed: resection of the only right kidney under pharmaco-cold anti-ischemic protection (Fig. 6); stopping bleeding; elimination of hemotamponade of the collecting system; plastic pelvis with local tissues; internal drainage stent placement.

Histology: clear cell RCC with foci of necrosis and hemorrhage, with foci of invasive growth in its own capsule pTibNoMo.

Then the patient underwent regular US monitoring of the state of the right kidney.

10 months after surgery, another US examination revealed an echodense lesion, $52.4 \times 28.4 \mathrm{~mm}$, with fairly clear contours at the hilum of the kidney in the pelvis (Fig. 7). The collecting system distal to this zone was expanded (up to $11.7 \mathrm{~mm}$ ). The vascular pattern of the kidney outside the above-described zone was evenly expressed. The ureter at the hilum of the kidney was expanded to $11.9 \mathrm{~mm}$, its lumen was free. Conclusion. Condition after resection of the right kidney. Expansion of the renal collecting system due to a partial block at the pelvis, most likely caused by a hematoma.

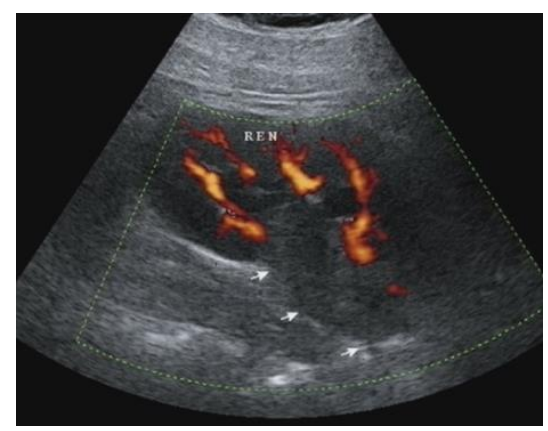

Figure 7. US, EDI, expansion of the renal (REN) collecting system due to a partial block at the pelvis, most likely caused by a hematoma (arrows)

The patient underwent MRI. Conclusion. Condition after resection of the only right kidney. The lesion in the lumen of the renal pelvis, closely surrounded by the branches of the renal artery, causing a moderate violation of the passage of urine (Fig. 8). In terms of signal characteristics, the lesion may correspond to an organized hematoma.

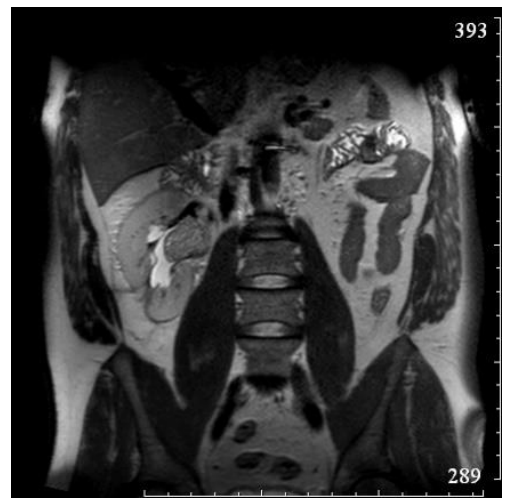

Figure 8. MRI, T2W_TSE, expansion of the renal collecting system due to the lesion in the pelvis

According to the data of US-monitoring, an increase of the lesion was noted.

The patient was recommended to undergo an MSCT. In the lumen of the renal pelvis, the lesion of an irregular rounded shape, with lobed contours was

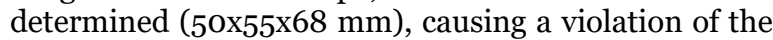
outflow of urine and a blurred expansion of the kidney cups. The density of the lesion in the native phase was $44 \mathrm{HU}$ (Fig. 9a), the accumulation of contrast agent occurs in the arterial phase (up to $110 \mathrm{HU}$ ) (Fig. 9b) along the lower and external contours, the impression of the presence of a leg emanating from the area of postoperative changes, and small supplying arterial vessels, traced also from the area of postoperative 
changes. Excretion of contrast agent by the kidney is delayed (Fig. 9 c,d). Conclusion. MDCT-signs of the pelvis mass lesion of the right kidney, recurrence in the area of surgical intervention cannot be ruled out. Hydronephrosis on the right, delayed excretion of contrast agent by the kidney.
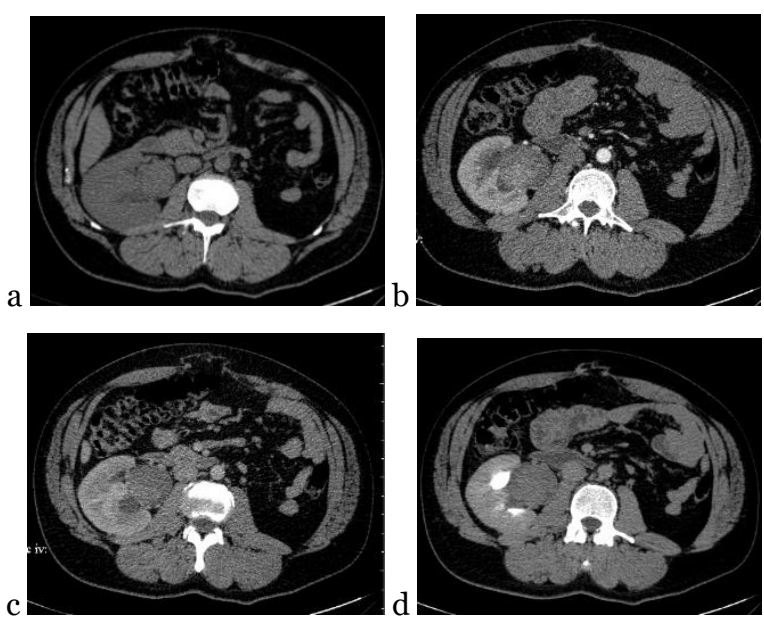

Figure 9. MDCT of the pelvis lesion of the right kidney in the native (a), arterial (b), venous (c) and delayed (d) phases
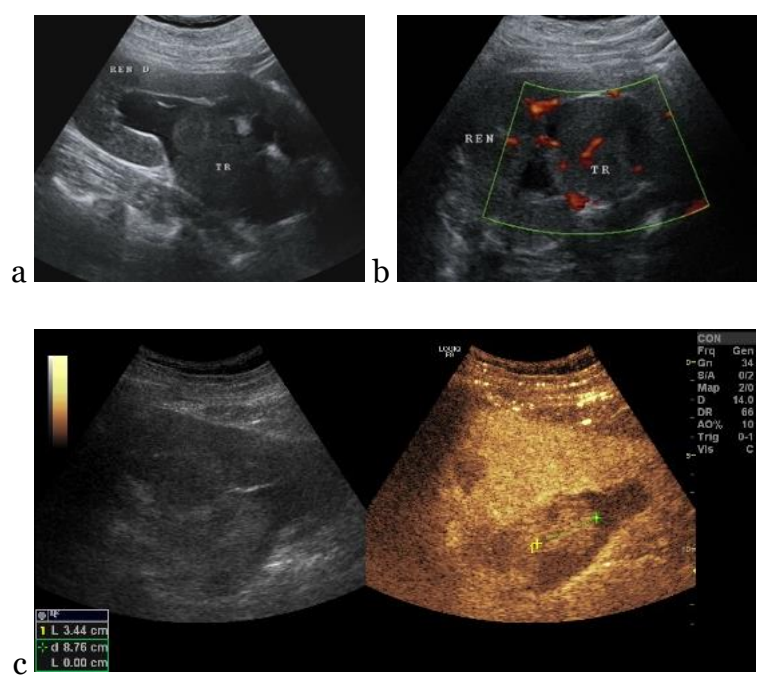

Figure 10. US of a tumor thrombus in the renal pelvis: a tumor thrombus (TR) in the renal pelvis (REN D) (B-mode); b - zone of fixation of a tumor thrombus (TR) to the renal pelvis (REN) with the presence of thrombus vascularization in this zone (EDI); e - the nature of Sonovue accumulation in tumor thrombus indicates the presence of blood flow only in the pole, fixed to the resection zone (indicated by labels)

The data obtained indicated the recurrence of the tumor of the only right kidney.

The patient was hospitalized for surgery.

To determine the zone of active growth of the lesion with the aim of clearer and faster intraoperative navigation, US was performed. The lesion was fixed to the zone of kidney resection and it was seems that it was supplied with blood from this zone (vascularization is traced only in this pole) (Fig. 10 a,b). After Sonovue injection at the pole, where vascularization is determined, local accumulation of the contrast agent (up to $20.0 \mathrm{~mm}$ zone) occurs in the arterial phase of the study, followed by its washing out (Fig.10c).

Surgery was performed: resection of the only right kidney under pharmaco-cold anti-ischemic protection; resection of the lower group of cups; elimination of tamponade of the collecting system; right urinary tract stenting; right-sided nephrostomy; plastic pelvis with local tissues; aortocaval lymphadenectomy.

Intraoperatively. The lesion was contoured in the projection of the kidney pelvis, its diameter was up to 6 $\mathrm{cm}$. Visually, the pelvis was filled with tumor masses along its entire length. The lesion with fairly clear contours in the pelvis of the kidney was determined at IOUS (Fig. 11a). The lesion was fixed to the zone of kidney resection and is supplied with blood vessels from this zone (vascularization is traced only in this pole) (Fig. 11b). Access to the tumor through the area of the previous resection was determined under US-control (Fig. 12a). The renal artery and vein were clamped. Manita solution $100 \mathrm{ml}$ was injected intravenously. The kidney is immersed in a crumb of ice. The renal artery and two veins are clamped with Bulldog vascular forceps. At a distance of $0.5 \mathrm{~cm}$ from the tumor, the right kidney parenchyma was resected. The tumor masses were evacuated from the lumen of the pelvis (Fig. 12 b,c). The site of fixation of the tumor node with its excision (the region of the lower cups) was visualized (Fig. 12d). The pelvic defect is sutured. The blood flow through the vascular system of the right kidney was restored. Heat renal ischemia $50 \mathrm{~min}$. The vascular pattern in the kidney is quite evenly expressed at IOUS, in the resection zone it is somewhat depleted. In the dilated pelvis, hypoechoic blood clots are determined (the lumen isn't obturated), as well as the proximal end of the nephrostomy.
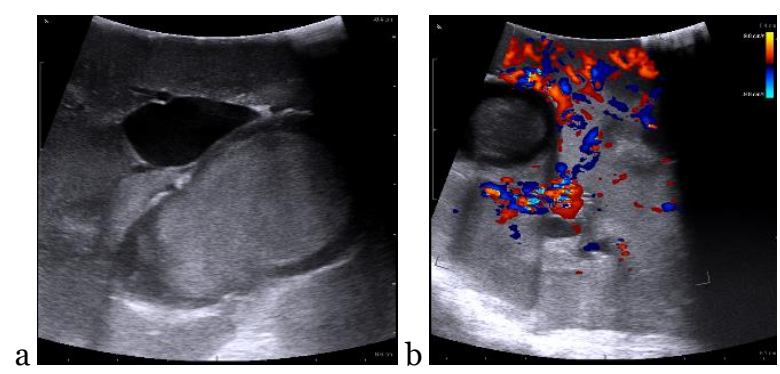

Figure 11. IOUS: a - thrombus in the renal pelvis, B-mode; $\mathrm{b}$ - increased blood supply to the resection area during the first surgery, to which a thrombus is fixed, CDI

The course of the postoperative period is smooth.

There was no fluid in the retroperitoneal space and in the small pelvis under US-control. The renal parenchyma in the area of resection had a nonuniformly reduced echogenicity with echodense inclusions. Renal collecting system was enlarged due to the pelvis $(21.1 \mathrm{~mm})$, the contents of which were heterogeneous due to hypoechoic clots; the proximal end of the nephrostomy and ureteral stent was traced in its cavity. The vascular pattern in the resection area was somewhat depleted; in other parts of the kidney, it was evenly and fairly pronounced. RI in all parts of the segmental arteries was within the normal range, in the resection area was $0.54-0.6$. 

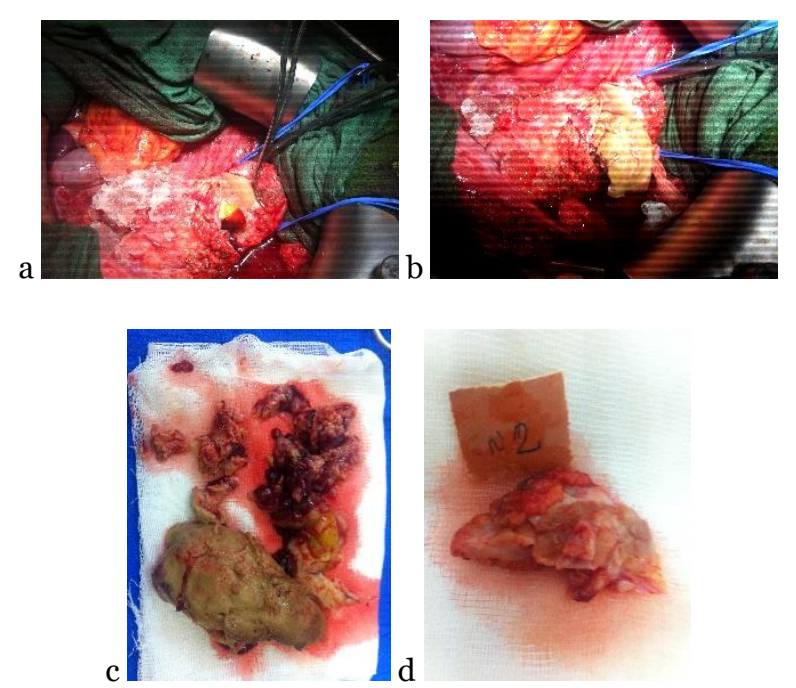

Figure 12. Intraoperative photos: a - blood-supplied "head" of a tumor thrombus; $\mathrm{b}$ - tumor thrombus turned out of the pelvis; $c$ - tumor thrombus; $d$ - a removed fragment of the renal pelvis wall in the zone of thrombus fixation

The above changes returned to normal with dynamic monitoring.

The patient underwent targeted therapy in the postoperative period. Against this background, after 1 month, the nephrostomy was removed. There were no data obtained for distant metastasis.

The presented clinical case illustrates the possibilities of various radiology methods, especially US, in the primary and clarifying diagnosis of cancer of a single kidney and recurrence of the disease in its pelvis, as well as the monitoring of the results of organpreserving surgical treatment of a patient with a single kidney.

The analysis of data from the patients treated at A.V. Vishnevsky NMRC of Surgery showed high efficiency of US at the stages of extracorporeal renal resection. Based on our own experience in the treatment of such patients, an algorithm for the use of various modalities of US in the pre-/intra- and postoperative periods had been developed.

The results of our study indicate that the US effectiveness is especially high in specialized clinics that deal with oncourology and have modern diagnostic US equipment with a wide range of modalities. However, we should remember that MDCT and/or MRI can also provide accurate information about the level of tumor spread, especially in cases of limited echographic imaging due to the patient's constitutional characteristics or poor preparation for the study.

\section{CONCLUSION}

Extracorporeal resection of a solitary kidney is a technically difficult intervention, requiring not only precise surgical technique, but also a specific approach to examination and postoperative conservative therapy. This operation should be performed in a selected group of patients in a highly specialized multidisciplinary hospital. The results obtained are satisfactory, which indicates the advisability of further development of organ-saving treatment.

Radiology methods play a crucial role in the determination of treatment tactics, the scope of surgery, as well as dynamic control. Active use of US various modalities at monitoring allows to control the patient's condition and to apply adequate methods of its correction in time. Moreover, US don not bear any radiation load and do not use nephrotoxic contrast agent, which is of special significance for the patients with RCC. Furthermore, it is possible to repeat the study several times and evaluate it in real time, and this study is also cheaper than MDCT and MRI.

Acknowledgements: The reported study was funded by RFBR according to the research project № 20-315-90107\20.

\section{REFERENCES}

1. Под ред. А.Д. Каприна, В.В. Старинского, Г.В. Петровой, Злокачественные новообразования в России в 2018 году (заболеваемость и смертность), М.: МНИОИ им. П.А. Герцена - филиал ФГБУ «НМИЦ радиологии» Минздрава России, 2019.

(A.D. Caprin, V.V. Starinskiy, G.V. Petrova, Eds. Malignant neoplasms in Russia in 2018 (morbidity and mortality), Moscow: Moscow P.A. Gertsen Research Institute of Oncology, 2019.)

Retrieved from

https://glavonco.ru/cancer register/\%Do\%97\%Do\%B O\%Do\%B1\%Do\%BE\%Do\%BB 2018 \%Do\%AD\%Do\% BB\%Do\%B5\%D0\%BA\%D1\%82\%D1\%80.pdf

Retrieved on: Aug. 15, 2021

2. M. Tanaka et al., "Prognostic factors of renal cell carcinoma with extension into inferior vena cava", Int. $J$. Urol., vol. 15, no. 5, pp. 394-398, May 2008. https://doi.org/10.1111/j.1442-2042.2008.02017.x

3. T.K. Choueiri et al., "Updated efficacy results from the JAVELIN Renal 101 trial: first-line avelumab plus axitinib versus sunitinib in patients with advanced renal cell carcinoma", Ann. Oncol., vol. 31, no. 8, pp. 1030-1039, Aug. 2020. https://doi.org/10.1016/j.annonc.2020.04.010

4. E. Shapiro, D.A. Goldfarb, M.L. Ritchey, "The congenital and acquired solitary kidney", Rev Urol., vol. 5, no. 1, pp. 2-8, 2003. PMID: 16985610 PMCID: PMC1472993

5. E. Tantisattamo et al., "Current Management of Patients with Acquired Solitary Kidney", Kidney International Reports, vol. 4, no. 9, pp. 1205-1218, 2019. https://doi.org/10.1016/j.ekir.2019.07.001

6. P.K.-T. Li et al., "Kidney Health for Everyone Everywhere - From Prevention to Detection and Equitable Access to Care", Kidney Diseases, vol. 6, no. 3, pp. 136-143, 2020. https://doi.org/10.1159/000506528

7. S. Groen in't Woud, L. van der Zanden, M.F. Schreuder, "Risk stratification for children with a solitary functioning kidney", Pediatric Nephrology, vol. 36, pp. 3499-3503, 2021.

https://doi.org/10.1007/s00467-021-05168-8

8. А.А. Теплов и др., «Метод экстракорпоральной резекции почки в условиях фармако-холодовой ишемии без пересечения мочеточника с ортотопической реплантацией сосудов при почечноклеточном раке», Экспериментальная $u$ клиническая урология, № 2, с. 52-62, 2015. (A.A. Teplov et al., "The method of extracorporeal resection of the kidney in conditions of pharmaco-cold 
Yu.A. Stepanova et al., Ultrasound ... single kidney extracorporeal resection..., RAD Conf. Proc., vol. 5, 2021, 141-147

ischemia without crossing the ureter with orthotopic replantation of vessels in renal cell carcinoma", Experimental and Clinical Urology, vol. 2, pp. 52-62,

2015.)

Retrieved from: https://readera.org/142188379

Retrieved on: Aug. 15, 2021 